\title{
Homeostatic model assessment (HOMA) in relation to lipid profiles and vitamin D status in South Asian and Caucasian women: preliminary results from D-FINES
}

\author{
O. A. Hakim ${ }^{1}$, K. Hart $^{1}$, A. L. Darling ${ }^{1}$, F. Shojaee-Moradie ${ }^{1}$, J. L. Berry ${ }^{2}$, A. M. Umpleby ${ }^{1}$, \\ B. A. Griffin ${ }^{1}$ and S. A. Lanham-New ${ }^{1}$ \\ ${ }^{1}$ Nutrition and Metabolism Department, Faculty of Health and Medical Sciences, University of Surrey, Guildford GU2 7XH \\ and ${ }^{2}$ Vitamin D Research Group, University of Manchester M13 9WL, UK
}

It is well recognised that populations with the most insulin resistance are at greater risk of developing cardiovascular disease and metabolic syndrome ${ }^{(1)}$. However little is known about the UK-based South Asian population in comparison to their Caucasian counterparts. This subsidiary study is part of the D-FINES study (Vitamin D, Food Intake, Nutrition and Exposure to Sunlight in Southern England). This secondary analysis aimed to quantify insulin resistance (IR), beta cell function (\%B), and insulin sensitivity (\%S) using the homeostatic model assessment (HOMA) in both ethnic groups and to investigate if they were any associations with vitamin D status $25(\mathrm{OH}) \mathrm{D}$ and lipid profiles.

The HOMA calculator from the University of Oxford (Diabetes Trails Unit, 2004) was used to estimate IR, \% B and \%S using fasting glucose and insulin level ${ }^{(2)}$. The analysis was from summer 2008 and included 185 Caucasian and 52 South Asian women aged 19-70 years old. We have previously shown that South Asian women had a significantly lower $25(\mathrm{OH}) \mathrm{D}$ status $(p<0.001)$ and a significantly higher BMI than the Caucasian group $(p<0.01)$. Table 1 below presents the $\% \mathrm{~B}, \% \mathrm{~S}$ and IR means for both ethnic groups. South Asian were shown to have significantly higher beta cell function and lower insulin sensitivity $(p<0.05, p<0.001$ respectively) than the Caucasian group. South Asians were significantly more insulin resistant than the Caucasian group $(p<0.001)$.

\begin{tabular}{lcc} 
Table 1. HOMA model among Caucasian and South Asian women & Asian \\
\hline beta cell function $(\% \mathbf{B})$ & Caucasian & $121.02(50.68)$ \\
insulin sensitivity $(\boldsymbol{\%})$ & $103.18(33.48)^{*}$ & $54.12(25.16)$ \\
IR & $72.06(35.24)^{* * *}$ & $2.29(1.42)$ \\
\hline$* * * p<0.001, * * p<0.01, * p 0.05$, all variables were not normally distributed analysed by Mann-Witney $\mathrm{U}$.
\end{tabular}

HOMA variables did not correlate with $25(\mathrm{OH}) \mathrm{D}$ status for either ethnic groups. However, beta cell function correlated positively with mean total cholesterol and low density lipoprotein (LDL) among South Asian females $(p<0.001)$. In this group there was also a significant positive correlation between insulin resistance and mean triglyceride (TAG) $(p<0.01)$. All correlations were adjusted for BMI.

Lack of association between the HOMA model and 25(OH)D status among the South Asian populations may due to the year-long, severe vitamin D deficiency in this group. However the lipid correlation findings indicate that South Asian women are at high risk of cardiovascular disease with respect to the lipid profile associations with the HOMA model. Further investigations are certainly warranted.

1. McFarlane et al. (2001) J Clin Endocrinol Metab 86, 713-718.

2. HOMA calculator. Oxford: diabetes trail unit, University of Oxford. 